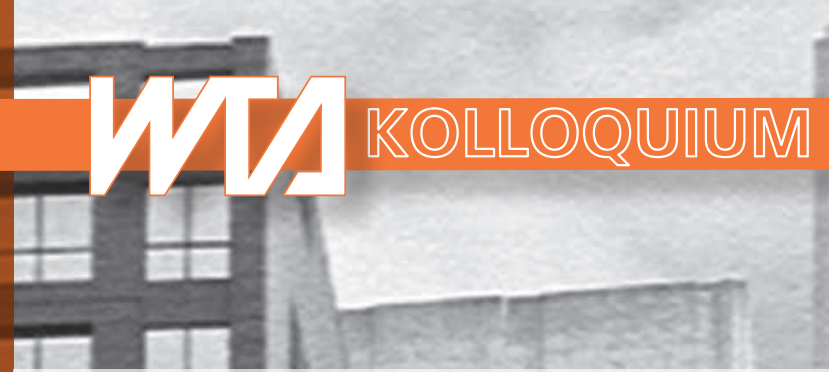

Hans-Peter Leimer (Hrsg.)

\title{
Bauinstandsetzen und Bauphysik
} gestern - heute - morgen

\section{Restauration and} Building-Physics past - present - future
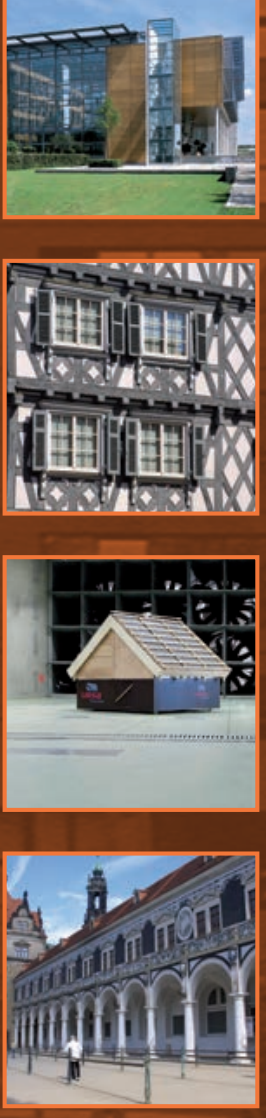

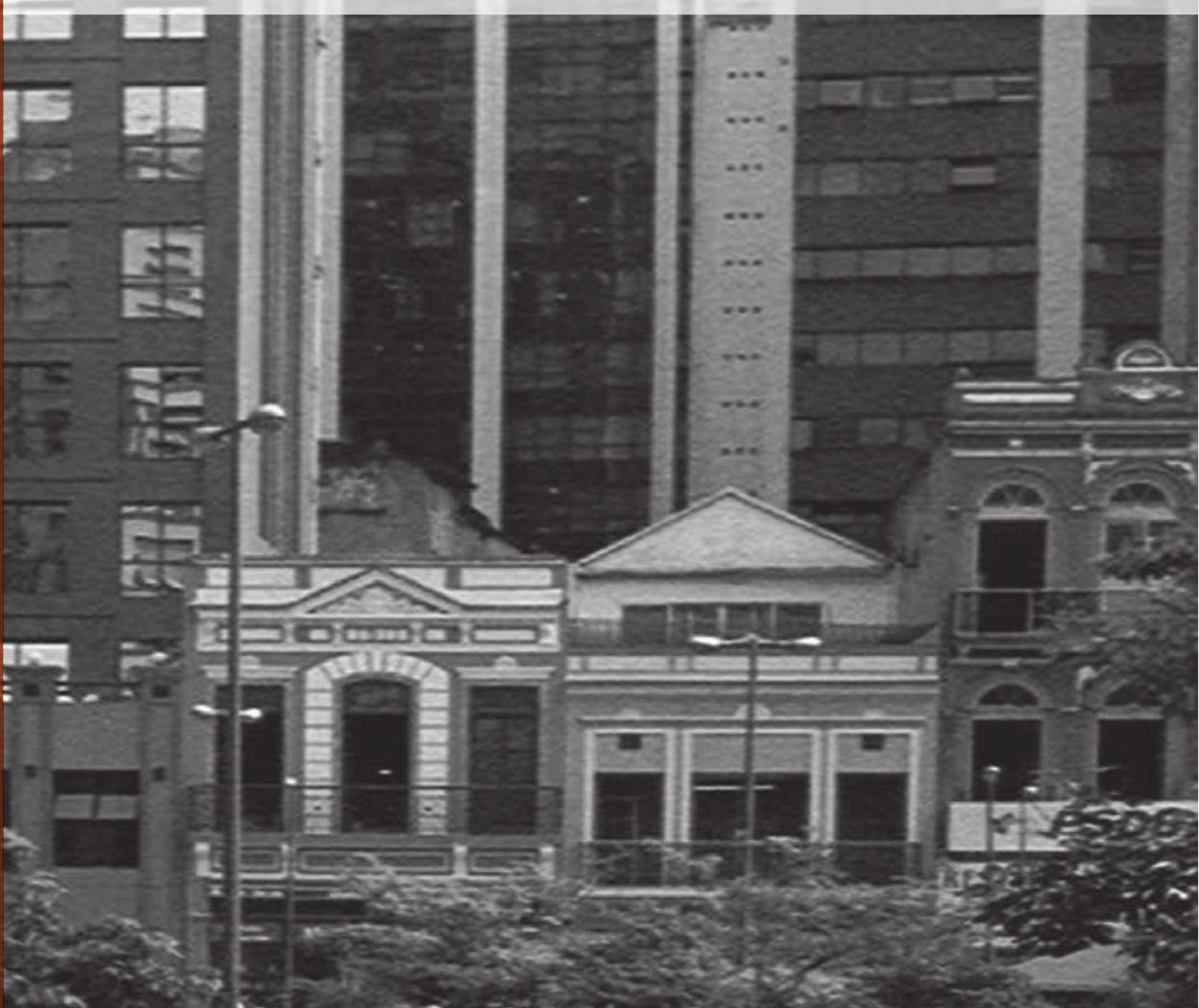

Fraunhofer IRB Verlag

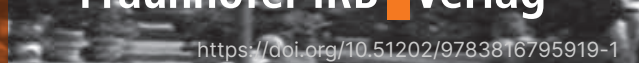

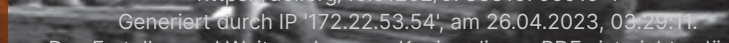
Das Erstellen und Weitergebenvon.Konion dieses PDFs ist nichtzulässig.

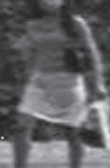


Hans-Peter Leimer (Hrsg.)

Bauinstandsetzen und Bauphysik gestern - heute - morgen

Restauration and Building-Physics past - present - future 
Hans-Peter Leimer (Hrsg.)

\section{Bauinstandsetzen und Bauphysik gestern - heute - morgen}

\section{Restauration and Building-Physics past - present - future}

32. Internationales WTA-Kolloquium 
Bibliografische Information der Deutschen Nationalbibliothek:

Die Deutsche Nationalbibliothek verzeichnet diese Publikation in der Deutschen Nationalbibliografie;

detaillierte bibliografische Daten sind im Internet über www.dnb.de abrufbar.

ISBN (Print): 978-3-8167-9590-2

ISBN (E-Book): 978-3-8167-9591-9

32. Internationales WTA-Kolloquium Bauinstandsetzen + Bauphysik VII

10. und 11. März 2016

HAWK Hochschule für angewandte Wissenschaft und Kunst

Goschentor 1

31134 Hildesheim

Herstellung: Andreas Preising

Layout: Fraunhofer IRB Verlag

Umschlaggestaltung: Martin Kjer

Satz: Fraunhofer IRB Verlag

Druck: Westermann Druck Zwickau GmbH, Zwickau

Alle Rechte vorbehalten.

Dieses Werk ist einschließlich aller seiner Teile urheberrechtlich geschützt. Jede Verwertung, die über die engen Grenzen des Urheberrechtsgesetzes hinausgeht, ist ohne schriftliche Zustimmung des Fraunhofer IRB Verlages unzulässig und strafbar. Dies gilt insbesondere für Vervielfältigungen, Übersetzungen, Mikroverfilmungen sowie die Speicherung in elektronischen Systemen.

Die Wiedergabe von Warenbezeichnungen und Handelsnamen in diesem Buch berechtigt nicht zu der Annahme, dass solche Bezeichnungen im Sinne der Warenzeichen- und Markenschutz-Gesetzgebung als frei zu betrachten wären und deshalb von jedermann benutzt werden dürften.

Sollte in diesem Werk direkt oder indirekt auf Gesetze, Vorschriften oder Richtlinien (z. B. DIN, VDI, VDE) Bezug genommen oder aus ihnen zitiert werden, kann der Verlag keine Gewähr für Richtigkeit, Vollständigkeit oder Aktualität übernehmen. Es empfiehlt sich, gegebenenfalls für die eigenen Arbeiten die vollständigen Vorschriften oder Richtlinien in der jeweils gültigen Fassung hinzuzuziehen.

(C) Fraunhofer IRB Verlag, 2016

Fraunhofer-Informationszentrum Raum und Bau IRB

Nobelstraße 12, 70569 Stuttgart

Telefon $+49711970-2500$

Telefax $+49711970-2508$

irb@irb.fraunhofer.de

www.baufachinformation.de
Wissenschaftlich-Technische Arbeitsgemeinschaft für Bauwerkserhaltung und Denkmalpflege e. V. (WTA) WTA - Geschäftsstelle Ingolstädter Str. 102, 85276 Pfaffenhofen

Telefon +498957869727

Telefax +498957869729

wta@wta.de

www.wta.de 


\section{Vorwort Prof. Dr.-Ing. John Grunewald}

Es ist wieder so weit. Es trifft sich, wer Rang und Namen hat, zum 32. internationalen WTA-Kolloquium am 10. und 11. März 2016 in Hildesheim.

Das Thema »Bauinstandsetzen+Bauphysik« des diesjährigen WTA-Kolloquiums ist von grosser Bedeutung für einen sehr wichtigen Teil unserer Umwelt - unseren Gebäudebestand. Die enge Verbindung von Energieeffizienz, Klimawandel und Gesundheit mit unserem Gebäudebestand mag auf den ersten Blick nicht offensichtlich erscheinen, doch die Referate nationaler und internationaler Expertinnen und Experten werden diese Zusammenhänge eindrücklich aufzeigen.

Nach einer Einführung zum Thema »Entwicklung der Bauphysik im Spannungsfeld von Bautradition und Bauforschung « und der Diskussion über »Restoration of Concrte Structures « und »Conservation of Built Heritage in China « wird spezifischen Fragen hinsichtlich der »Bestandsaufnahme und neuen Anforderungen and Planungswerkzeuge« nachgegangen. Im weiteren Verlauf des zweitägigen WTA-Kolloquiums widmen sich die Vortragenden vielen spannenden und hochaktuellen Themen des Bauinstandsetzens und der Bauphysik.

Dem Gastgeber des Kolloquiums, unserem lieben Kollegen und Freund Herrn Prof. Dr.-Ing. Dipl.-Arch. Hans-Peter Leimer, dem wir anläßlich seines 60. Geburtstages herzlich gratulieren und viel Gesundheit und viele Erfolge wünschen, ist es maßgeblich zu verdanken, dass sich dieses Gebiet so erfolgreich entwickelt hat. Er hat es sich zur Lebensaufgabe gemacht, die wissenschaftlichen Grundlagen, besonders auf den Gebieten der Bauwerkserhaltung, der Bauphysik und der Bauchemie aufzunehmen und in Handlungsanweisungen für die Praxis zu überführen. Durch seine Forschungstätigkeit als Institutsleiter an der Hochschule für angewandte Wissenschaft und Kunst HAWK Hildesheim und der Hefei University in China und die langjährige Leitung des BBS Ingenieurbüros hat er in hervorragender Weise dazu beigetragen, den Dialog zwischen Wissenschaft, Fachleuten und der Praxis zu fördern.

Das 32. internationale WTA-Kolloquium wird vielfältige Impulse für Universitäten, Forschungsinstitute, Behörden, Architekten, Ingenieure, Unternehmen und Verbände geben. Während der Konferenz bieten sich so auch viele Gelegenheiten, sich mit internationalen Expertinnen und Experten und Kolleginnen und Kollegen auszutauschen.

Prof. Dr.-Ing. John Grunewald 


\section{Vorwort Prof. Dr.-Ing. Harald Garrecht}

2016 ist für die Wissenschaftlich-Technische Arbeitsgemeinschaft für Bauwerkserhaltung und Denkmalpflege ein Jahr mit zwei herausragenden Momenten. So kann der Verein im Oktober 2016 auf sein 40-jähriges Bestehen zurückblicken. Dieses besondere Ereignis wird im Herbst mit einer Festveranstaltung in München gewürdigt werden. Doch schon im Vorfeld dieses Jubiläums hat die WTA einen weiteren Grund, ein großes Ereignis zu feiern, wie die festliche Aufmachung des Konferenzbandes zum „32. Internationalen WTA-Kolloquium Bauinstandsetzen+Bauphysik VII“, das anlässlich der WTA-Tage 2016 in Hildesheim veranstaltet wird, verrät.

Anfang März 2016 feiert unser langjähriger und ehemaliger 1. Vorsitzende, Prof. Dr.Ing. Hans-Peter Leimer, seinen 60. Geburtstag. Das Erweiterte WTA-Präsidium hat sehr gerne seiner Bitte entsprochen, die WTA-Tage 2016 in Hildesheim stattfinden zu lassen und die Organisation und Durchführung des Kolloquiums in die Hände von Hans-Peter Leimer zu geben. Dies ermöglicht allen der WTA zugehörigen und verbundenen Gäste, mit dem Jubilar anstoßen zu können.

Vor 24 Jahren wurde Hans-Peter Leimer Mitglied in der WTA. Von Beginn an brachte er sich mit großem Engagement in der Referatsarbeit ein. Natürlich hat er sich über die Facharbeit hinaus auch für die Fortentwicklung der WTA insgesamt und die Verwirklichung der Vereinsziele unermüdlich eingesetzt. Als die WTA 1996 in unruhige Fahrwässer geriet, wurde er 1997 in den Vorstand gewählt. Dort übernahm er die wichtige Aufgabe, Strategien zu entwickeln, um den Verein zu konsolidieren und nicht die erfolgreiche fachlich-inhaltliche Arbeit der Referate zu gefährden. 2001 wurde er dann zum 1. Vorsitzenden der WTA gewählt. Unter seiner Führung konnten die Strukturen der WTA verbessert und Regularien geschaffen und gelebt werden, dank deren sich die Mitglieder heute wieder in ihren Referaten und ihren Regionalen Gruppen gerne engagieren. Auch stellte er wichtige Weichen, den Verein fortan nicht rein national, sondern multinational auszurichten. 2013 schied Hans-Peter Leimer dann nach zwölfjährigem weithin sichtbarem Wirken aus dem WTA-Vorstand aus. Für seine vielfältigen Leistungen wurde ihm 2013 in Braunschweig die Ehrenmitgliedschaft in der WTA zuerkannt. Sein Wirken als 1. Vorsitzender prägte die WTA in den zurückliegenden Jahren. Doch wirken sich die Entwicklungen der vergangenen Dekade auch auf die bevorstehende Vereinsentwicklung aus.

Hans-Peter Leimer hat in seiner Zeit als 1. Vorsitzender der WTA ein gut funktionierendes und weit verzweigtes Netzwerk entwickelt, wie der Inhalt des vorliegenden Konferenzbandes verdeutlicht. So ist es ihm gelungen, über 63 Referentinnen und Referenten für das 32. Internationale Kolloquium zu gewinnen, um in den adressierten Themenfeldern „Bauinstandsetzen und Bauphysik“ beizutragen. Wichtig war ihm, all jene Forschungen und Entwicklungen aufzugreifen und zu streifen, die den Stand der Bautechnik bis in die Gegenwart beeinflussten. Doch sollen die Beiträge nicht nur zurückblicken. Vielmehr soll die Veranstaltung auch einen Ausblick gewähren, um den Teilnehmerinnen und Teilneh- 
mern wie auch den Leserinnen und Lesern die Möglichkeit zu bieten, die Chancen und Perspektiven ihres eigenen Wirkens zu reflektieren.

Freuen wir uns also darauf, in Hildesheim mit Hans-Peter Leimer seinen 60. Geburtstag zu feiern und an der informationsreichen und anregenden Konferenz teilzunehmen. Dem Jubilar wünschen wir auch für die nächsten Jahre ein weiterhin erfolgreiches Schaffen und Wirken.

Stuttgart, Februar 2016

Prof. Dr.-Ing. Harald Garrecht 


\section{Vorwort Prof. Dr.-Ing. Klaus Sedlbauer}

Der Abschluss einer jeden Dekade bietet eine gute Gelegenheit zu feiern, aber auch auf das Vergangene zurückzublicken. Mit der kontinuierlichen Weiterentwicklung der Bautechnik, der Bauphysik, der Fortschreibung der gesetzlichen Normen und Verordnungen sowie der Weiterentwicklung des Standes von Wissenschaft und Technik, bietet das Bauwesen ein herausforderndes Umfeld für jeden, der im Bauwesen ingenieurmäßig, forschend oder lehrend tätig ist. Und besonders Hans-Peter Leimer ist in allen drei Tätigkeitsfeldern sehr aktiv. Ingenieurmäßig durch die Begleitung vieler nationaler und internationaler Bauprojekte wie zur Expo 2010 in Shanghai mit dem Pavillion of Innovations. Im Bereich der Bauforschung seien beispielsweise seine Aktivitäten im Bereich der energetischen Gebäudesimulation genannt, welche zu dem Low Energy Vertificate LEC als Planungs- und Optimierungstool zur energetischen Bewertung von Gebäuden führten. Im Bereich der Lehre verstärkte Hans-Peter Leimer seine Aktivität vor allem im internationalen Bereich durch die Professur für das Lehrgebiet Bauphysik an der Hefei Universität in China. In Verbindung mit seiner Lehrtätigkeit in China steht auch der Aufbau des Instituts für Angewandte Bauphysik und Dauerhaftigkeit. Im Fokus steht hier die Prüfung und Zertifizierung von Bauprodukten sowie die baubegleitende Qualitätssicherung in China. Vor dem Hintergrund der Häufigkeit von umfangreichen Bauschäden, gerade im Neubau, ist in China eine solche Einrichtung dringend erforderlich. Aber auch seinen bisherigen Schwerpunktthemen der Bauinstandsetzung und der Denkmalpflege widmete sich Hans-Peter Leimer in dem Projekt zur Entwicklung eines Instandsetzungskonzeptes unter städtebaulichen, architektonischen und bauphysikalischen Gesichtspunkten für die Stadtvillen Bao Yang in Shanghai, China. Innerhalb dieses Projektes konnte er gut auf die Normen und Regelwerke der WTA zurückgreifen, deren Präsident er von 2001 bis 2013 war.

Entsprechend dem Motto des 32. Internationalen WTA-Kolloquiums „Forschungen und Entwicklungen, die die Bautechnik beeinflussten, ein Rückblick - ein Ausblick“ bleibt ein kurzer Blick in die Zukunft. Diese wird durch den aktuellen Umbruch in weiten Teilen der Welt stark geprägt sein. Ein großer Impuls in Richtung Neubauprojekte kommt durch die Zuwanderung an Flüchtlingen. Auch wird das Bauwesen stark beeinflusst durch die derzeit extrem fallenden Ölpreise. Diese machen energieeffizientes und ökologisches Bauen unattraktiver. Insbesondere diejenigen, die sich mit Bauinstandsetzung intensiv auseinandersetzen wissen, dass Gebäude sehr langlebige Objekte sind und kurzfristige Trends fast immer überstehen. Daher wird es aus meiner Sicht immer wichtiger, den kompletten Lebenszyklus des Gebäudes zu beachten und hierbei den Mensch als Nutzer nicht zu vergessen.

Es bleibt an dieser Stelle noch Hans-Peter Leimer für die kommenden Jahre beste Gesundheit zu wünschen, so dass er in der Bauinstandsetzung und der Bauphysik sowohl als Ingenieur, als Architekt, als Bauforscher und als Professor weiter wirken kann.

Prof. Dr.-Ing. Klaus Sedlbauer 


\section{Vorwort Prof. Dr.-Ing. Andreas Holm}

Hans-Peter Leimer wird am 7. März 2016 sechzig Jahre alt. Er hat mit seinem Wirken in besonderer Weise zur Steigerung der Energieeffizienz bei gleichzeitiger Erhöhung der Wohnqualität beigetragen. Das Jahr 2016 ist aber auch für die Energiewende ein besonderes Jahr. Das Energieeinsparungsgesetz des Bundes (EnEG) wird 40 Jahre alt. Mit seinen vielen wissenschaftlichen Arbeiten aber auch seiner Praxisnähe über sein Ingenieurbüro, das er seit 25 Jahre führt, hat er maßgeblich zum Gelingen der Energiewende beigetragen.

Bis in die 1960er und 1970er Jahre bestand eine Außenwand aus 36,5 cm Ziegel- oder Leichtbetonmauerwerk mit etwas Putz auf der Außen- und Innenseite und vielleicht noch ein wenig Farbe zur Verschönerung. In Zeiten scheinbar unendlich verfügbarer Energie war an energieeffiziente Gebäude nicht zu denken. Die niedrigen Energiepreise sorgten selbst in einem strengen Winter für mollig warme Häuser. Der in schon den 1950er Jahren eingeführte Mindestwärmeschutz diente nicht der Energieeinsparung, sondern stellte die Tauwasser- und Schimmelfreiheit der Oberflächen der Außenbauteile sicher. Mit dem Ö1preisschock Mitte der 1970er Jahre begann dann der Umdenkprozess. Man erkannte, dass die Gebäude für einen großen Teil des Energieverbrauchs in Deutschland verantwortlich waren.

Das Energieeinsparungsgesetz des Bundes (EnEG) in seiner ersten Fassung von 1976 war Grundlage der ersten Wärmeschutzverordnung von 1977. Das EnEG zielt darauf ab, in Gebäuden Energie zu sparen und nur so viel Energie zu verbrauchen, wie jeweils notwendig ist um das Gebäude zweckdienlich zu nutzen. Es hatte schon in der ersten Fassung insbesondere den Wärmeschutz der Gebäudehülle sowie die effiziente Anlagentechnik und deren Betrieb im Visier. Daraufhin wurden erste Anforderungen an den Wärmeschutz neu zu errichtender Gebäude eingeführt. Die Einführung der ersten Wärmeschutzverordnung 1978 und die in den späteren Jahren erfolgten Anpassungen sowie die Einführung der EnEV 2002 hat in Deutschland den Neubau von 1,75 Mrd. m² Wohnfläche (ca. 40\% der gesamten Wohnfläche) beeinflusst. Die energetische Qualität der Gebäude hat seit der Wärmeschutzverordnung 1979 deutlich zugenommen und ist inzwischen um ein vielfaches besser als die vonm Vorkriegsaltbauten. Ohne diese, auch schon damals, immer wieder umstrittenen Anpassungen würde der Energieverbrauch für Raumwärme und Warmwasser im Wohngebäudebestand heute jährlich 250 TWh höher liegen. Das heißt, anstatt derzeit knapp 575 TWh würden deutsche Wohngebäude jährlich fast $825 \mathrm{TWh}$ an Energieverbrauch aufweisen.

Die von der Bundesregierung im Energiekonzept 2050 formulierten Zielvorgaben bei der Reduzierung des Primärenergiebedarfs sind klar. Um die avisierte Verminderung des Primärenergieverbrauchs um 50\% gegenüber 2008 zu erreichen, soll der Primärenergieverbrauch im Gebäudebereich um $80 \%$ reduziert werden. Dieses ambitionierte Ziel ist allein mit einer energieeffizienten Ausführung von Neubauten nicht zu erreichen. Es bedarf also in jedem Fall einer planvollen Sanierung des Gebäudebestands unter Ausnutzung aller 
sinnvollen aktiven (Haustechnik) und passiven (Dämmung) Maßnahmen. Vergleicht man die Gebäudebestandsverteilung mit dem entsprechenden energetischen Zustand der Gebäude, so stellt man fest dass derzeit $65 \%$ der Gebäude in Deutschland sanierungsbedürftig sind. Der Anteil nachträglich gedämmter Außenflächen von Altbauten liegt bei Ein- und Zweifamilienhäusern bei ca. $20 \%$, bei Mehrfamilienhäusern bei ca. $26 \%$. Fast die Hälfte aller Dachflächen, aber nur ein sehr geringer Teil der Kellerdecken in Altbauten wurden nachträglich gedämmt. Ausgehend von einem technisch realisierbaren Einsparpotenzial ergibt sich im gesamten Gebäudebereich (Wohn- und Nichtwohngebäude) ein grob geschätzter Minderverbrauch von ca. 350 bis $400 \mathrm{TWh}$ pro Jahr, also die fast vierfache Leistung aller deutschen sich momentan in Betrieb befindenden Atomkraftwerke. Diese theoretische Effizienzverbesserung ist natürlich nur abrufbar, wenn bei allen Gebäuden sämtliche Möglichkeiten, wie eine ausreichende Dämmung der Gebäudehülle, eine Fenstermodernisierung und der Einsatz moderner Technik, vollständig genutzt werden.

Gebäude und Räume müssen heute neben Anforderungen an den Wärmeschutz selbstverständlich auch Anforderungen an die energetisch und akustisch sinnvolle Raumkonditionierung erfüllen.. Und hier zeigt sich auch welche Bedeutung die Bauphysik für die Energiewende in den letzten Jahrzehnten hatte bzw. haben wird. Denn neben den Fragen des Wärmeschutzes sind es auch die des Feuchte-, Schall- und Brandschutz die die Wünsche und Anforderungen von Bewohner an die Behaglichkeit und die Gesundheitsverträglichkeit unserer Gebäude beeinflussen. In der Praxis zeigt sich jedoch, dass der Einhaltung dieser Maßgaben von Bauherren und Investoren zu wenig Aufmerksamkeit gewidmet wird. Das erkannte Hans Hans-Peter Leimer sehr früh und hat mit seinem Wirken in besonderer Weise zur Steigerung der Energieeffizienz bei gleichzeitiger Erhöhung der Wohnqualität beigetragen. Er war einer der ersten Bauphysiker die unter anderem modernen Rechenverfahren in der Praxis einsetzten. Er berechnete die bauphysikalischen Vorgänge in Bauteilen und Gebäude instationär und trug durch sein Engagement im Rahmen der WTA dazu bei das sich die Verfahren sich immer mehr gegenüber den in DIN 4108 beschriebenen Normverfahren durchsetzen. Bei der diagnostischen Beurteilung der Feuchteverhältnisse legte er bei seinen Objekten wegweisen Wert darauf die verschiedene Einflüsse wie Schlagregen, aufsteigende Grundfeuchte oder Baufeuchte in Bauteilen entsprechend zu berücksichtigen. Er hat damit das energiesparende Bauen in Deutschland maßgeblich geprägt und gefördert. Wir gratulieren ihm herzlich zum 60ten Geburtstag und wünschen ihm weiterhin die Gesundheit und Kraft sein großes Engagement im beruflichen Alltag weiterzuführen.

Prof. Dr.-Ing. Andreas Holm 


\section{Vorwort Prof. XIA Yong}

Mentor und Freund: Im Jahr 2005 lernte ich Herrn Prof. Dr. Hans-Peter Leimer kennen. Bald schon wurden wir gute Freunde.

Ich meine, dass er wirklich mein Mentor und ein hilfreicher Freund ist. Nicht nur, weil er nach China viele Informationen über Energieeinsparung bei Gebäuden und die Anwendung der erneuerbaren Energien im Bauwesen mitbrachte, sondern auch unseren Studierenden und Dozenten die praktischen Lehrmethoden bei der Vorlesung Bauphysik aufzeigte und mit uns verschiedene interessante Workshops unternahm. Die Studierenden fühlten immer seine Gewissenhaftigkeit, Ernsthaftigkeit und Disziplin.

Zehn Jahre der Zusammenarbeit vergingen so schnell, doch habe ich die lange Zusammenarbeit noch immer vor meinen Augen, sie ist in meinen Kopf geblieben.

Schon im Jahr 2006 gründeten die Universität Hefei und die HAWK Hildesheim mit der Hilfe von Prof. Leimer einen neuen Bachelor-Studiengang Bauingenieurwesen 2+3 (Jahre), an dem die chinesischen Studierenden nach einem 2-jährigen Vorstudium an der HAWK in Hildesheim weiter studieren können.

Im Januar 2008 hielt Herr Prof. Leimer vor unseren Studenten die erste Vorlesung Bauphysik, die, erstmalig bei uns, als Modul in das Curriculum integriert wurde. Bis heute besucht Prof. Leimer die Deutsche Hochschule Hefei mehrmals im Jahr, um Vorlesungen auf den Gebieten der Bauphysik und des Energieeinsparens zu halten. Sein chinesisches Lehrbuch der Bauphysik ist nicht nur für die Studierenden hilfreich, sondern auch für ganz die Ausbildung in China, da es das Erste auf diesem Gebiet ist.

In den vergangenen Jahren führten wir gemeinsam mit Prof. Leimer nahezu jedes Jahr Workshops in China durch. Workshops waren eine neue Lehrmethode für uns. So erhielten wir im Juni des Jahres 2007 durch Herrn Prof. Leimer die Einladung, um an einem Workshop in Nanjing teilzunehmen. Die Universität Tongji sowie die Universität Südost Nanjing, die HAWK und die Universitäten Stuttgart und München, nahmen an diesem Workshop teil. Das Thema des Workshops waren die Planungen zur Energieeinsparung an einem bestehenden Gebäude. Hierzu diente das Lehrgebäude Qiangongzuan auf dem Campus der Universität Südost Nanjing als Vorgabe. Durch diesen Workshop hatten wir die erste Gelegenheit die Energieeinsparungsplanung in Deutschland zu verstehen. Neben der Projektarbeit an einem realen Gebäude lernten unsere Studierenden die praktische Anwendung in Gruppenarbeit. Diese Veranstaltung gab den Studenten der verschiedenen Kulturen die Gelegenheit sich kennenzulernen und Gedanken auszutauschen. Dieses erlebten wir auch auf der deutschen Ausstellung mit dem Thema „Deutschland und China - gemeinsam in Bewegung, Nanjing 2007 - Stadtvisionen“.

Im Mai 2014 unternahmen wir einen Workshop in die Provinz Shanxi/China, um an ausgewählten Dachsystemen von Tempelanlagen der Ming Dynasty in der Shanxi Provinz eine Schadenserfassung und Schadensanalyse durchzuführen. Grundlage der Untersuchungen war der Hui Ji Tempel nahe der Stadt Yuanping. Erbaut wurde der Hui Ji Tempel in der 
Tang-Dynastie (618-907) und wurde in der Song-Dynastie (960-1279) wieder aufgebaut, während der der Yuan-Dynastie (1271 - 1368) und der Qing-Dynastie (1644-1911) mehrmals renoviert. Wir erfuhren in diesem Workshop wie und was an alten Gebäuden untersucht werden muss, um sie sinnvoll restaurieren zu können.

Unser nächster Workshop im Mai 2015 führte uns in das Museumsdorf Qiankoh in der Huangshan Region der Provinz Anhui. Es gibt hier viele alte Häuser der Ming und Qing Dynastie (1368-1644). In den 80iger Jahren wählte die Regierung zehn verschiedene, in der nahen Umgebung gelegene, alte Gebäude aus, trug sie ab und errichtete sie wieder in Qiankoh. Die alten Gebäude stellen die verschiedenen Kulturen und die Geschichte in der jeweiligen Zeit dar und bestehen aus drei Tempeln, vier Wohnhäusern, einem steinernen Tor, einer steinernen Brücke sowie einem Pavillon. Bei diesem Workshop erhielten die Studenten die Aufgabe der Entwicklung eines Ausstellungskonzeptes für das gesamte Museum sowie eine raumklimatische Untersuchung für ein Museumsgebäude. Weiterhin sollten Planungsgrundlagen zur Instandsetzung anhand eines Beispielgebäudes erarbeitet werden.

Die Energieeinsparung von Gebäuden wurde in den 90iger Jahren in China neu geregelt. Aus diesem Grund empfahl Herr Prof. Leimer eine Plattform für Technologietransfer zur Qualitätssicherung im Bauwesen in der Provinz Anhui aufzubauen. Diese Plattform wurde am 10. Oktober 2008 in Hefei von der Universität Hefei Anhui - China, dem Bauministerium der Provinz Anhui, dem Bauministerium der Stadt Hefei, dem Prüfinstitut der Provinz Anhui für Bauqualität, dem Prüfinstitut für Bauqualität der Stadt Hefei und dem Prüfinstitut der Produktionsqualität der Provinz Anhui gegründet.

Über diese Plattform fanden und finden Vorträge für Hochschulen und Institute, für Bauunternehmen und für Architekten statt. Dieses führte dazu, dass die Kontakte zwischen Behörden und Bauunternehmen, Universitäten und Prüfungsinstitute deutlich ausgeweitet werden konnten. Besonders hervorzuheben ist auch der neue intensive Kontakt zwischen den Institutionen der Provinz Anhui, den deutschen Universitäten und deutschen Unternehmen.

Die EXPO 2010 Shanghai war ein wichtiger Meilenstein, bei dem Prof. Leimer den Pavillon of Innovations mit mehr als 50 Projektpartnern, auch mit der Hefei Universität, in Shanghai errichten konnte, um aktuelles deutsches Know-how anlässlich der Weltausstellung zu präsentieren. Durch den großen Erfolg hoffen wir, dass wir ein vergleichbares Gebäude durch Herrn Prof. Leimer auf unserem Campus in kurzer Zeit errichten können.

Aufgrund des Beitrags und der Leistungen von Herrn Prof. Leimer für die Zusammenarbeit zwischen dem Land Niedersachsen und Provinz Anhui hat die Regierung der Provinz Anhui ihm 2009 den Huangshan-Freundschaftspreis verliehen.

Wir hoffen sehr, dass Herr Prof. Leimer und die Hefei Universität auch in Zukunft die Zusammenarbeit der beiden Länder und beider Hochschulen weiter pflegen und intensivieren können.

Prof. XIA Yong 


\section{Inhaltsverzeichnis}

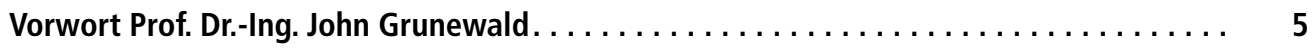

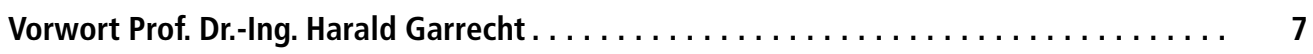

Vorwort Prof. Dr.-Ing. Klaus Sedlbauer. $\ldots \ldots \ldots \ldots \ldots \ldots \ldots \ldots \ldots \ldots \ldots \ldots \ldots$

Vorwort Prof. Dr.-Ing. Andreas Holm $\ldots \ldots \ldots \ldots \ldots \ldots \ldots \ldots \ldots \ldots \ldots \ldots \ldots \ldots$

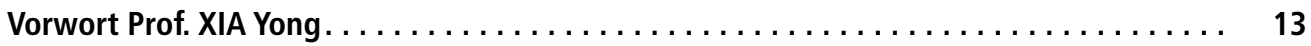

Keynotes

Die Entwicklung der Bauphysik im Spannungsfeld von Bautradition und Bauforschung . . 23

Klaus Peter Sedlbauer

Development in the Rehabilitation of Concrete Structures in the Czech Republic

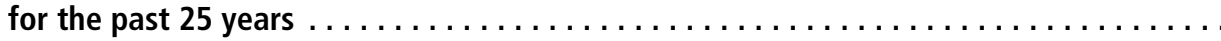

Rostislav Drochytka

Planungswerkzeuge für Energieversorgungskonzepte in Gebäuden und Quartieren . . . . .

Bestandsaufnahme und neue Anforderungen

John Grunewald

Conservation of Built Heritage in China $-\ldots \ldots \ldots \ldots \ldots \ldots \ldots \ldots \ldots \ldots \ldots \ldots \ldots \ldots \ldots \ldots$

with a Focus on Material Conservation

Shibing Dai, Gesa Schwantes

Feuchte- und Salzbeanspruchung von Mauerwerk $\ldots \ldots \ldots \ldots \ldots \ldots \ldots \ldots \ldots \ldots$

Verständnis früher und heute

Harald Garrecht

Thermische Ergonomie: Ein Rückblick auf die wichtige Rolle der Bauphysik

Historische Entwicklung und aktuelle Trends

Christoph van Treeck, Jérôme Frisch, Caroline Lorz, Henning Metzmacher,

Maximilian Praster, Carolin Schmidt, Daniel Wölki, Sebastian Wolf

Dämmstoffe - Eine Entwicklungsgeschichte

Andreas Holm 


\section{Bauinstandsetzen}

Nachhaltige Bauwerkserhaltung und Denkmalpflege - ein modernes Phänomen . . . . 133 Susanne Mühlhaus

Structural Intervention Strategies for Monuments Constructed in

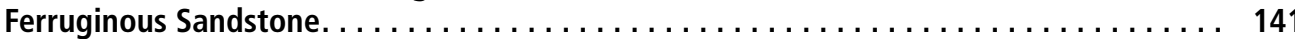

Three Belgian case studies

Els Verstrynge, Dionys Van Gemert

Wärmeschutz und Energieeinsparung bei Bestandsbauten

Alles im Fluss - aber nicht ohne Mäander!

Frank Eßmann

Einsatz von Bauradar zur Erkundung von Mauerwerks- und Betonbauwerken

Gabriele Patitz

Mauerwerksinstandsetzung und hydrophobe Imprägnierung

Rudolf Plagge, Philipp Heinze

Konservierungswissenschaftliche Begleitung der Inbetriebnahme einer innovativen Lüftungsanlage in Schloss Linderhof

Kristina Holl, Stefan Bichlmair, Katrin Janis, Tina Naumovic', Ralf Kilian

Nachhaltigkeit in der Kulturerbeforschung - Langzeiterfahrungen mit der Untersuchung historischer Gebäude .

12 Jahre konservierungswissenschaftliche Forschung in der Kapelle St. Renatus in Lustheim

Ralf Kilian, Stefan Bichlmair, Kristina Holl, Susanne Raffler, Robert Krah,

Martin Krus, Klaus Sedlbauer

Putze als Multitalent

Sylvia Stürmer

Trockenmörtel im Wandel der Zeit .

Speziallösungen im Bereich der energetischen Innensanierung

Heike Pfaff

Spritzbarer, säurebeständiger Epoxidharzmörtel.

Eine Neuentwicklung für die Instandsetzung von Abwasserbauwerken aus Beton unter

Beanspruchung mit biogener Schwefelsäure

Robert Schulte Holthausen, Heiner Stahl, Michael Raupach

Hydrophobierung von Werkstoffen des Bauwesens.

Technologietransfer von der Antike in das 21. Jahrhundert

Matthias Schwotzer, Julia Süßmuth, Andreas Gerdes 
Innendämmung. $\ldots \ldots \ldots \ldots \ldots \ldots \ldots \ldots \ldots \ldots \ldots \ldots \ldots \ldots \ldots \ldots \ldots \ldots \ldots \ldots \ldots \ldots \ldots \ldots, 257$

Eine vielfach verkannte Geschichte

Anatol Worch

Vernagelung von Gewichtsmauern

Standsicherheitsnachweise und Langzeitverhalten

Erwin W. A. Schwing

Visible and hidden qualities of matter's documentary value in historic buildings .......

Elena Zapatero-Rodriguez

3D-Fotogrammetrie - neue Dokumentationsmethoden zur Grundlagenermittlung ......

Andreas Bruschke

Raumklima in Magazinräumen.

Konzept und Umsetzung einer interdisziplinär angelegten Risikoanalyse für Archiv- und Bibliotheksgut Johanna Kraemer

Entwicklung von Ingenieur- und Forschungsmodellen zur Vorhersage der Dauerhaftigkeit nachträglicher Verfugungen in historischem Mauerwerk ..

Carolin Westermann, Heiko Twelmeier, Heinrich Wigger, Harald Garrecht

Ermittlung der Tragfähigkeit von Ziegelbrücken gestern - heute - morgen .

Viktor Bartolomei, Heinrich Wigger

Hygrometrische Feuchtemessung - Forschung, Entwicklung und Anwendung in der Praxis ...

hygrometric moisture measurement - research, developement and application

Dennis Ziegler, Uwe Schürger

Nachträgliches Abdichten erdberührter Bauteile im Wandel der Zeit

Wilhelm Fix

Kathodischer Korrosionsschutz von Stahlbeton. .

Ein smartes Instandsetzungsverfahren mit Zukunft

Felix Wenk

Ultrahochleistungs-Faserbeton in der Schweiz - von der Idee bis zur bewährten Lösung . . 375 Peter Lunk, Kerstin Wassmann

Von der Betoninstandsetzung zur Betoninstandhaltung ...

Erforderliche Maßnahmen im Sinne des zukünftigen WTA-Merkblatts Instandhaltung und der

DAfStb-Richtlinie Instandhaltung von Betonbauteilen.

Rolf P. Gieler 
Stil-Ikone mit FOAMGLAS ${ }^{\circledast}$ Wärmedämmung für Lifestyle und Zukunftssicherung revitalisiert...

Dirk Vogt

Zerstörungsfreie Analyse von Instandsetzungsmaßnahmen mittels NMR. .

Jeanette Orlowsky

\section{Bauphysik}

Von der hygrothermischen Bauteilsimulation zum Risikomanagement in der Bauphysik. .

Thomas Bednar

Der Energieausweis - ein Erfolgsmodell !?

Ulrich Möller

Entwicklung mineralischer Baustoffe für Neubau und Bestand.

Wenn aus Vorhersagen Wirklichkeit wird: Werkstoffe zum Bauen im 21. Jhdt.

Jürgen Gänßmantel

Die Entwicklung der Bioenergie im Wandel der Zeit .

Von reiner Wärmeerzeugung bis hin zum flexiblen Einsatz als Systemdienstleister

in modernen Energieversorgungssystemen

Achim Loewen

Flachglas - früher und heute .

Die Entwicklung und Anwendungen von Flachglas von seinen Anfängen bis heute

Reinhard Cordes

Raumakustik und Beschallung in halligen Räumen

Mathias Krumbiegel

Vom Abriss gewachsener Stadtstrukturen in Shanghai hin zu neuen Nutzungskonzepten. .

Der Wandel in der Wahrnehmung alter Gebäudestrukturen am Beispiel der Kreativparkgelände

in Shanghai

Frank Krüger, Pascal Hartmann

Numerische Methoden in der hygrothermischen Bauteilsimulation

Entwicklungsschritte und Stand-der-Technik

Andreas Nicolai

Modellierung hygrothermischer Materialeigenschaften für numerische

Simulationsverfahren in der Bauphysik

Eine Entwicklung von den Anfängen bis heute

Gregor A. Scheffler 
Raumklima im Wandel - Raumklimatische Untersuchungen in Callcenter . . . . . . 517 Hans-Peter Leimer, J. Schneider

Entwicklung von Nutzungsprofilen unter soziologischen Gesichtspunkten . . . . . . 525 Neue Ansätze zur Vorhersage des Nutzerverhaltens

Jan Schneider

Nachhaltigkeit

Jürgen Engel, Oliver G. Hamm (Red.)

Funktionelle Gipsplatten

Moderne Baustoffe mit Zusatznutzen

Hans-Ulrich Hummel

Bauphysik und Leichtbau

Leichtes Bauen und darauf abgestimmte Bauphysik

bereichern das Bauen in Deutschland

Harald Hünting

Entwicklung der Fensteranschlussfuge unter Betrachtung mittels Berechnungs-,

Simulations- und Nachweisverfahren.

Björn Kethorn

Rückblick auf 25 Jahre diffusionsoffene Dachbahn für das Steildach

Reiner Thiele, Co-Autor: Hans-Peter Leimer

Sustainability in the Chinese Buliding Sector

Hans-Peter Leimer

Kulturelle Einflussfaktoren beim Bauen und bei der Bauinstandsetzung in China

Roland Winkler 
